\title{
Estabelecendo Processos de Software em Micro e Pequenas Empresas
}

\author{
Sérgio Weber ${ }^{1}$, Jean Carlo Rossa Hauck ${ }^{2}$, Christiane Gresse von Wangenheim ${ }^{3}$ \\ ${ }^{1}$ Universidade Federal de Santa Catarina - UFSC \\ Programa de Pós-Graduação em Ciência da Computação \\ Florianópolis/SC - Brasil \\ ${ }^{2}$ VOID CAZ Sistemas Ltda \\ Florianópolis/SC - Brasil \\ ${ }^{3}$ Universidade do Vale do Itajaí - UNIVALI \\ Laboratório de Qualidade e Produtividade de Software - LQPS \\ São José/SC - Brasil \\ weber@inf.ufsc.br, jean@voidcaz.com.br, gresse@univali.br
}

\begin{abstract}
This paper proposes an approach for establishing software processes in small companies aiming at the improvement of product quality and productivity. The paper describes the principal steps of the approach and indicates guidelines for its application in the context of this specific type of organization. The approach is being developed principally based on our experiences of defining and implementing software processes in small software companies in Florianópolis/SC. First experiences, as well, as results analyzing the applicability and cost/benefit relation of the approach are also presented.
\end{abstract}

Resumo. Este artigo propõe uma abordagem para estabelecer processos de software em micro e pequenas empresas, ajudando a melhoria da qualidade dos produtos e da produtividade. $O$ artigo descreve os principais passos da abordagem e indica diretrizes para sua aplicação no contexto específico desse tipo de organização. A abordagem está sendo desenvolvida baseada principalmente na experiência adquirida em definição e implantação de processos de software em micro e pequenas empresas em Florianópolis/SC. Primeiras experiências, bem como resultados analisando a aplicabilidade e a relação custo/benefício da abordagem também são apresentadas.1

\section{Introdução}

De acordo com dados do Ministério da Ciência e Tecnologia [19], o mercado nacional na área de software é marcado pela predominância de micro e pequenas empresas (MPEs) $)^{2}$. Contudo, a taxa de mortalidade dessas organizações é bastante elevada e

\footnotetext{
${ }^{1}$ O presente trabalho foi realizado com o apoio do Programa Art. 170 do governo do estado de Santa Catarina e do CNPq, uma entidade do governo brasileiro voltada ao desenvolvimento científico e tecnológico.

${ }^{2}$ Considerando micro e pequenas empresas (MPEs), empresas com até 49 funcionários.
} 


\section{Simpósio Brasileiro de Qualidade de Software}

segue uma tendência também observada em outros setores. Uma pesquisa realizada pelo SEBRAE [28], por exemplo, mostra que 49,4\% das empresas morrem até os dois primeiros anos de existência, aumentando para 56,4\% até os três primeiros anos e $59,9 \%$ até os quatro primeiros anos. Dentre os principais fatores que contribuem para esses dados estão a forte concorrência, a inexperiência gerencial e de marketing dos proprietários, a alta carga tributária, a falta de capital de giro e a ausência de políticas internas voltadas para a garantia da qualidade dos produtos. Tais fatores geram uma série de dificuldades em MPEs, tornando-as enfraquecidas para competir no mercado.

Um caminho que contribui para que uma organização se torne mais competitiva e cresça é investir na melhoria da qualidade e da produtividade. Como a melhoria da qualidade do produto final é tipicamente atingida através da melhoria do próprio processo produtivo, melhorar os processos de software é um desafio para a indústria brasileira de software. Em particular, MPEs têm geralmente um processo de software informal e, consequentemente, dependente principalmente da competência das pessoas envolvidas [19]. Nesse contexto, o estabelecimento sistemático de processos pode contribuir significativamente na sua melhoria e assim, aumentar sua competitividade e suas chances de sobrevivência. Para estabelecer um processo, o mesmo precisa ser definido e implantado. Ao definirmos um processo é preciso que seja construído um modelo que o represente. Essa representação suporta o entendimento e a visualização do processo, facilita sua disseminação e comunicação, auxilia na gerência de projetos, e é importante na avaliação, evolução e melhoria contínua do processo.

Para a modelagem de processos, existem várias abordagens [1]. No entanto, a maioria delas está focada em representações formais, voltadas para execução automática do processo, ou para empresas de grande porte. Refinando as abordagens existentes e enfocando especificamente nas características de MPEs (comunicação simples, processos instáveis, necessidade de agilidade, inexperiência na área de engenharia de software, recursos limitados, etc.), nós começamos a desenvolver uma abordagem para estabelecimento de processos de software voltada para empresas desse porte. Essa abordagem está baseada na adaptação dos modelos e abordagens existentes na literatura [5] [17] [24], em experiências anteriores com modelagem de processo de software realizados pelo LQPS/UNIVALI e pelo Grupo de Qualidade de Software do Centro GeNESS [8] [9] [30] [31], e em outras experiências de terceiros [18] [23].

\section{Estabelecimento de Processo de Software}

A qualidade dos produtos de uma empresa é proveniente da qualidade do processo pelo qual o mesmo foi concebido. Um processo é uma seqüência de passos realizados para um determinado propósito [11]. Um processo de software é definido como um conjunto de atividades, métodos, práticas e transformações que pessoas empregam para desenvolver e manter software e produtos associados [21]. O processo de software é um processo complexo que pode ser subdividido em sub-processos, incluindo, além dos processos técnicos (p.ex. codificação e testes), também processos gerenciais (p.ex. gerência de projetos e da qualidade) e organizacionais (p.ex. melhoria de processos, treinamento). A norma ISO/IEC 12207 [12], por exemplo, divide o processo de software em três categorias e vinte e dois sub-processos.

Para poder prover melhoria no processo de software de uma organização, os mesmos devem estar bem caracterizados e compreendidos. Para isso, um conjunto de 


\section{Simpósio Brasileiro de Qualidade de Software}

processos padrão deve ser definido e mantido em cada unidade da organização, juntamente com a construção de guias descrevendo sua aplicabilidade e orientando como customizá-los. Essas exigências, por exemplo, são requisitos obrigatórios para obter o nível 3 de capacidade dos modelos CMMI [29] e da norma ISO/IEC 15504 [13]. O processo padrão deve ainda ser utilizado por todos os projetos da unidade da organização, adaptando-o às características específicas dos projetos. Nesse contexto, é importante que a organização defina o seu processo de software por meio de alguma forma de representação. Essa representação pode ser feita de vários modos, principalmente como modelo de processos ou guia de processos [14].

Um modelo de processo de software é uma representação abstrata da arquitetura, projeto ou definição do processo de software, que descreve, em diferentes níveis de detalhes, uma organização dos elementos de um processo [1]. Um modelo de processo inclui, tipicamente, os seguintes elementos [1] [16] [32]:

- Atividade: é a etapa de um processo que produz mudanças de estado visíveis externamente no produto de software;

- Critérios de Entrada e de Saída: são condições que devem ser satisfeitas para que algo inicie (pré-condições) e para que algo termine (pós-condições);

- Papel: é um grupo de responsabilidades, privilégios e habilidades requeridas para executar uma atividade específica no processo de software;

- Produto: é um artefato associado com a execução de uma atividade, como, por exemplo, um caso de teste, uma especificação de requisitos ou o código;

- Ferramenta: é um instrumento de trabalho que auxilia na execução de um processo ou uma atividade;

- Método: é um conjunto de regras e critérios que estabelecem um caminho preciso e repetível de executar uma tarefa e encontrar um resultado desejado;

- Medidas: são descrições de uma característica de um determinado elemento do processo de forma qualitativa ou quantitativa.

Os principais modelos de referência, como ISO/IEC 12207 e CMMI, ou processos proprietários como RUP [22], especificam processos, atividades e tarefas de forma genérica. No entanto, não especificam como executá-los, nem levam em consideração o contexto específico de uma organização. Assim, modelar o processo dentro do ambiente em que ele será executado é bastante importante. Para isso, é preciso conhecer bem a organização envolvida (características, tipos de software que são desenvolvidos, paradigmas de desenvolvimento e cultura), de forma a conceber processos que atendam as suas necessidades, suas metas de negócio e contenham aspectos chaves da qualidade.

Em geral, a modelagem de processos pode ser executada de três formas: descritiva, prescritiva ou mista. A modelagem descritiva busca descrever como o processo é executado num contexto particular [5], buscando identificar a forma como as atividades são executadas dentro da organização. Por outro lado, a modelagem prescritiva busca descrever como o processo deveria ser executado [5], enfocando na melhoria do processo existente com base em best practices, em modelos de referência e normas da qualidade, como p.ex. a ISO/IEC 12207 ou CMMI. Outros tipos de modelagem buscam integrar descrição de processos com algumas prescrições que se fazem necessários. 
Durante muitos anos, a área de modelagem de processos se preocupou bastante com representações formais do processo, visando sua execução de forma automática. No entanto, os mesmos acabaram não sendo muito utilizados na prática, em razão das dificuldades em interpretar e disseminar um modelo formal [6] [7]. Nesse sentido, a forma de representar processos vem mudando o foco para construção de guias de processos. Um guia do processo é um documento de referência, estruturado para um processo particular, orientado a workflow, com o objetivo de dar suporte aos participantes do processo para que possam cumpri-lo [14]. Para isso, ele deve prover uma definição explícita do contexto e do processo ao qual se aplica, facilitando seu entendimento, comunicação e seguimento. Guias de processos podem ser documentados/armazenados em papel ou meios eletrônicos, estes últimos, chamados de Eletronic Process Guides (EPG). Além dos elementos de um modelo de processo, um EPG, tipicamente, contém: templates de documentos, avisos sobre erros geralmente cometidos, checklists das tarefas já realizadas, critérios de decisão, entre outros. EPGs geralmente são disponibilizados na intranet da empresa e têm se mostrado um poderoso instrumento de melhoria dos processos. Isso acontece, pois a visualização dos elementos do processo de forma gráfica e/ou textual, aliado a facilidade de navegação através de hyper-links, permite um melhor entendimento do processo. Além disso, a centralização do guia num único local facilita a atualização e evita problema com versões [14] [27]. Uma outra vantagem é a possibilidade de integrar um repositório de experiências como uma extensão para o EPG, o que permite capturar o feedback dos executores do processo em relação as atividades [26].

Vários trabalhos têm sido desenvolvidos na área de EPG’s, incluindo, por exemplo, [7] [14] [20] [26] e [27]. Existem também algumas ferramentas para EPGs, como, por exemplo, Spearmint [10] e WAGNER [25]. A primeira delas é uma ferramenta para modelagem que pode, automaticamente, gerar um EPG a partir de um modelo de processo definido. A segunda é um framework para gerar guia de processos para o ambiente WEB a partir de diagramas de atividade UML. Além dessas duas, outras ferramentas que suportam o trabalho cooperativo e o gerenciamento de workflows podem ajudar a organizar e facilitar o acesso a documentação do processo. Apesar da existência destes trabalhos na área de modelagem e construção de guias de processo, nenhum deles atende completamente os requisitos para estabelecimento de processos de software no contexto de MPE's: baixo investimento, fácil compreensão, orientação detalhada das atividades a serem executadas (como fazer), ser de domínio público, suporte de guias para sua implantação e adaptação, entre outros.

\section{Descrição da Abordagem ASPE/MSC ${ }^{3}$}

A abordagem para estabelecimento de processos de software em micro e pequenas empresas (ASPE/MSC) que estamos propondo foi desenvolvida com base em abordagens para modelagem de processos de software disponíveis na literatura e na nossa experiência na área. Para suportar o estabelecimento de processos de software de forma incremental, visando melhoria contínua, a abordagem está organizada em fases. Isso permite que a empresa possa estabelecer vários processos, um atrás do outro, ou,

\footnotetext{
${ }^{3}$ Sigla derivada da descrição em inglês: Approach for Software Process Establishment in Micro and Small Companies
} 
melhorar um único processo em vários ciclos, sempre acompanhando a sua aplicação e avaliando os resultados obtidos. Cada uma dessas fases principais, por sua vez, é dividida em sub-processos A figura 1 dá uma visão geral da abordagem e suas fases: diagnóstico do processo de software atual, análise estratégica, definição do processo e implantação do processo.

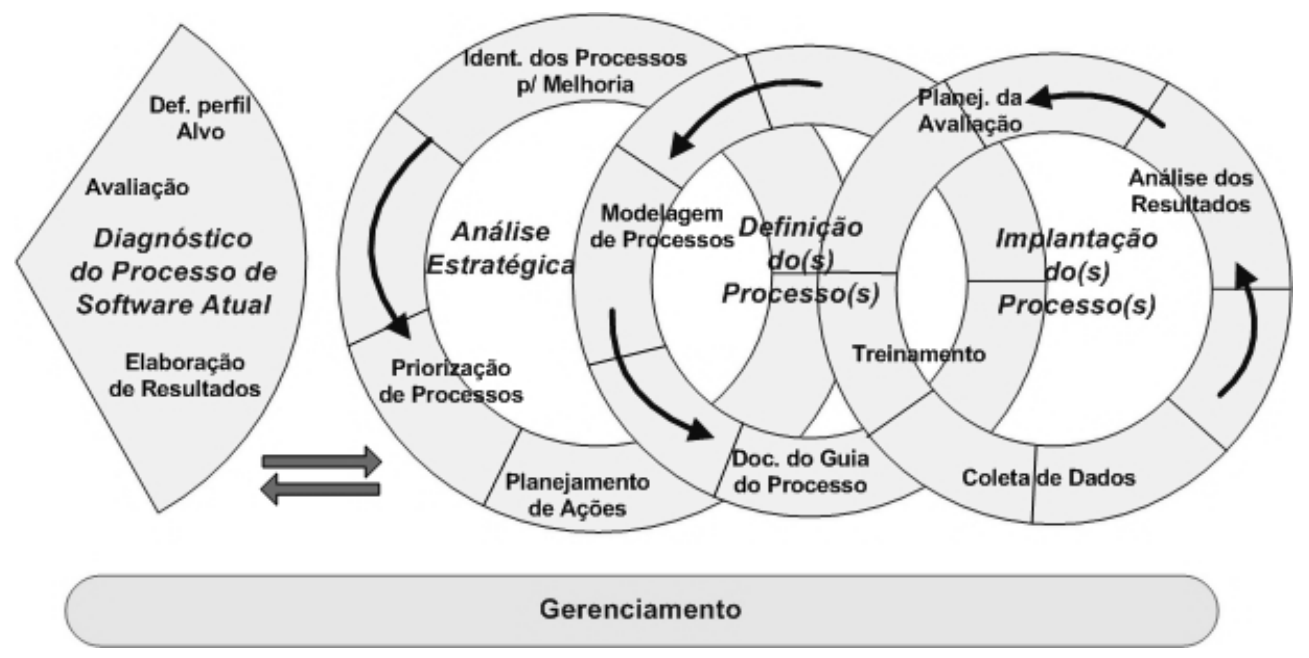

Figura 1: Visão geral da abordagem ASPE/MSC

O estabelecimento de processo de software é feito principalmente por um engenheiro de processo, que pode ser um consultor externo, caso a empresa não possua uma pessoa com competência nesta área. Além disso, é necessário o envolvimento, em tempo parcial, de um representante da organização (RO). O papel do RO é acompanhar como o engenheiro de processo aplica a abordagem na prática, com o objetivo de absorver competências. Além desses dois papéis, é necessário que a diretoria da empresa esteja comprometida com a aplicação da abordagem e atue constantemente na monitoria, motivação e controle. Os demais envolvidos no processo de software participam de todas as fases da aplicação da abordagem, principalmente quando envolvidos diretamente no(s) processo(s) sendo estabelecido(s). Todo o processo de estabelecimento é planejado, monitorado e controlado. Cada uma das fases da abordagem é descrita, a seguir, com mais detalhes.

\subsection{Fase 1 - Diagnóstico do Processo de Software Atual}

O objetivo da primeira fase é compreender, num nível macro, todo processo de software que está sendo executado na empresa. Nessa fase, oportunidades de melhoria são identificadas, os processos mais importantes em relação às metas de negócio da empresa e suas características específicas são priorizados, e uma visão geral da situação atual e dos processos da empresa é estabelecida. Isto pode ser alcançado por meio de uma avaliação do processo de software. Por exemplo, MARES - Metodologia de Avaliação de Processos de Software [2] [3], é um método de avaliação em conformidade com a norma ISO/IEC 15504 e adaptado especificamente para MPEs que pode ser utilizado para essa finalidade. Resultados de uma avaliação seguindo MARES incluem: 
- Descrição alto nível do processo de software da empresa, baseado no modelo de referência de processo da norma ISO/IEC 15504-5. A figura 2 dá um exemplo de um modelo de processo alto nível gerado.

\begin{tabular}{|c|c|c|c|c|c|}
\hline \multicolumn{6}{|c|}{$\begin{array}{l}\text { Gerenciamento de Projetos } \\
\text { (Planejamento de tarefas, cronograma e custos usando MSProject; monitoria e controle informal realizado semanalmente) }\end{array}$} \\
\hline Fornecimento & Desenvolvimento & Entrega & Instalação & Suporte & Manutenção \\
\hline $\begin{array}{l}\text { 1. Levantamento } \\
\text { das necessidades } \\
\text { do cliente } \\
\text { 2. Preparar } \\
\text { proposta } \\
\text { 3. Negociar e } \\
\text { preparar contrato }\end{array}$ & $\begin{array}{l}\text { 1. Análise de } \\
\text { Requisitos } \\
\text { 2. Implementação } \\
\text { 3. Testes } \\
\text { 4. Documentação } \\
\text { das classes e do } \\
\text { modelo de BD }\end{array}$ & $\begin{array}{l}\text { 1. Treinamento } \\
\text { (informal) } \\
\text { 2. Aceitação }\end{array}$ & $\begin{array}{l}\text { 1. Instalação } \\
\text { 2. Teste de Usuário } \\
\text { 3. Implantação }\end{array}$ & $\begin{array}{l}\text { 1. Registro da } \\
\text { requisição } \\
\text { 2. Classificação } \\
\text { da requisição } \\
\text { 3. Atendimento } \\
\text { da requisição } \\
\text { 4. ... }\end{array}$ & $\begin{array}{l}\text { 1. Novas } \\
\text { Funcionalidades } \\
\text { 2. Integração } \\
\text { com outros } \\
\text { sistemas de SW } \\
\text { 3. ... }\end{array}$ \\
\hline \multicolumn{6}{|c|}{$\begin{array}{c}\text { Gerenciamento de Documentos } \\
\text { Inclui manual do usuário, documentação do sistema (modelo de classes e BD), documentos internos, contratos não são gerenciad } \\
\text { sistematicamente, não há rotinas de backup sistemáticas }\end{array}$} \\
\hline \multicolumn{6}{|c|}{$\begin{array}{l}\text { Gerenciamento de Configuração } \\
\text { (Limitado a fontes usando a ferramenta CVS) }\end{array}$} \\
\hline
\end{tabular}

Figura 2: Exemplo da visualização de um modelo de processo Alto Nível

- Definição dos perfis-alvo de processo: identifica quais processos são mais importantes para a empresa e qual o respectivo nível de capacidade que eles devem possuir para que as metas de negócio e de melhoria sejam alcançadas. O método MARES usa uma adaptação da técnica SWOT [15] para classificar os processos e metas da empresa em mais/menos importantes e pontos fortes/fracos. Além disso, é feito uma estimativa dos custos necessários para melhorar cada processo, benefícios esperados e se há uma ordem/seqüência a ser considerado na melhoria, o qual será utilizado como critério de seleção para escolha dos processos a serem avaliados.

- Avaliação dos processos, onde são geradas observações referentes aos processos de software selecionados e a pontuação da sua capacidade. Além disso, são comparados os resultados dos perfis dos processos avaliados com os perfis alvo, identificando pontos fortes e fracos e oportunidades de melhoria.

Ao término dessa fase deve-se conhecer e ter documentado uma descrição alto nível do processo de software da organização, os perfis-alvo dos processos, os perfis avaliados, riscos, pontos fortes e fracos.

\subsection{Fase 2 - Análise Estratégica}

O objetivo desta fase é definir e priorizar ações para o estabelecimento de processos na empresa, tendo como base os resultados da fase de diagnóstico e de acordo com as metas de negócio e de melhoria da empresa. Caso já tenha sido encerrado um ciclo de estabelecimento de processo, os resultados obtidos também devem ser considerados.

O primeiro passo para definir o plano de ação é identificar, analisar e selecionar os processos a serem melhorados. Para isso, os processos que fazem parte do perfilalvo, identificados na fase de diagnóstico podem ser tomados como base. Na seqüência, é preciso priorizar os processos. Para manter o custo baixo e, considerando a infraestrutura típica disponível em uma MPE, poucos processos devem ser selecionados para serem estabelecidos em cada ciclo de melhoria. Para a priorização, deve ser levado em consideração: benefícios, custos estimados, interdependência entre processos, metas de negócio e melhoria, freqüência de uso do processo, grau de divergência entre seus 
executores, número de atores envolvidos, etc. Após a execução desse passo, os processos são agrupados em uma ordem decrescente de prioridade e o(s) mais prioritário(s) é/são escolhido(s) para a seqüência da aplicação da abordagem. A cada ciclo de melhoria, essa lista de prioridades deve ser revista: novos processos podem ser incluídos, outros podem ser excluídos e a ordem dos processos pode mudar. No passo seguinte, são definidas metas mensuráveis para o estabelecimento do(s) processo(s) escolhido(s) e um conjunto de ações é planejado para serem executadas na seqüência da aplicação da abordagem. Esse planejamento deve incluir a definição de um cronograma, alocação de recursos, riscos, milestones e o orçamento. O acompanhamento desse planejamento é tratado na fase de gerência da abordagem. Pelo caráter estratégico dessa fase, todas as suas atividades devem ser executas pela diretoria da empresa, em conjunto com o engenheiro de processo e o RO.

\subsection{Fase 3 - Definição de Processo(s)}

A fase de definição de processo(s) tem como objetivo definir um processo de forma explícita. Para isso, é preciso criar uma representação do mesmo e depois descrevê-lo de modo que as pessoas possam se orientar na execução das atividades.

Para que a cultura da organização não se perca, o ponto de partida é criar uma representação de como o processo é executado na prática, ou seja, modelar o processo de forma descritiva. Para isso, num primeiro passo procura-se identificar as principais atividades que são executadas durante o processo. A seqüência de execução que elas acontecem e os desvios condicionais existentes podem ser visualizados por meio de um fluxograma. O próximo passo é identificar os papéis e as responsabilidades relacionados a cada atividade contida no fluxograma. No terceiro passo, o objetivo é identificar os artefatos que são consumidos ou produzidos durante a execução de cada atividade, e, caso existam, seus respectivos templates. Na seqüência, é preciso identificar as medidas que devem ser coletadas durante a execução de uma atividade, pois estabelecem quais dados quantitativos e qualitativos serão coletados para suportar a gerência de projetos, a melhoria e a garantia da qualidade. Para completar a descrição do processo atual, é preciso levantar ainda outras informações importantes, como, por exemplo, critérios de entrada e de saída, métodos e ferramentas.

Para a execução dos passos anteriores, o engenheiro do processo e o RO, devem ter uma forte interação com os envolvidos no processo. Para isso, o processo modelado deve ser acompanhado e devem ser realizadas entrevistas e algumas reuniões de brainstorming, onde cada um poderá apresentar o processo sob a sua perspectiva e participar ativamente na definição do processo. Os resultados obtidos devem ser documentados pelo RO.

Uma vez modelado, um processo pode ter seu modelo completado ou adaptado, visando sua melhoria por meio de uma modelagem prescritiva. Para isso, é preciso identificar os pontos de melhoria no modelo. Esse passo deve ser executado pelo engenheiro de processo e pelo RO e estar baseado em necessidades apontadas pela diretoria e pelos participantes do processo. Para prover melhoria, o próximo passo é identificar alternativas, que podem incluir, por exemplo, mudanças no fluxo das atividades, inclusão de novas atividades, troca de métodos, adoção de ferramentas, entre outras. Possíveis alternativas podem ser identificadas com base em normas e modelos de referência de processo, como ISO/IEC 12207 ou CMMI, bem como em processos 


\section{Simpósio Brasileiro de Qualidade de Software}

proprietários como o RUP, ou ainda, em best pratices encontrados na literatura de engenharia de software. A seleção da(s) alternativa(s) deve levar em consideração as características da empresa e suas metas de negócio e melhoria. Após a seleção ter sido realizada, a(s) opção(ões) escolhida(s) deve(m) ser integrada(s) ao modelo, completando-o e atualizando-o.

A modelagem do processo provê apenas uma representação inicial do processo, tornando explícitos os principais elementos que o compõe. Essa representação precisa ser documentada pelo engenheiro de processo e revisada pelos participantes do(s) processo(s), com o objetivo de validá-la. A revisão busca detectar inconsistências, erros, elementos importantes que estão faltando, ambigüidades, entre outras. Baseado nessa revisão, a documentação inicial vai sendo atualizada e completada, até que todos os revisores a aprovem.

Apesar da documentação do modelo do processo prover uma boa orientação aos executores do processo, ela ainda não dá aos mesmos todo o suporte que eles precisam para executarem suas atividades. Assim, é preciso que seja feito um detalhamento da documentação do modelo e seja criado um guia do processo, tornando-o o mais explícito e detalhado possível. A criação do guia envolve, geralmente, a inserção de avisos sobre os erros mais cometidos, critério de decisão, experiências anteriores, descrição de metodologias e de técnicas utilizadas. Esse passo é de responsabilidade do engenheiro de processo e do RO. No entanto, devem ser realizadas revisões periódicas com os participantes do(s) processo(s), sendo que o guia só é dado como concluído quando todos os revisores tiverem-no aprovado.

Geralmente, o guia é desenvolvido em formato eletrônico e disponibilizado na intranet da empresa para que todos os participantes possam consultá-lo. Uma outra alternativa é usar um EPG para esta finalidade. MEPS [8] [9], por exemplo, é um protótipo de um sistema de software para a gerência de manuais de processos de software em micro e pequenas empresas. Há outros casos ainda, em que uma ferramenta é adaptada ou desenvolvida, baseando-se no guia, para suportar o processo.

\subsection{Fase 4 - Implantação do(s) Processo(s)}

O objetivo dessa fase é institucionalizar e avaliar o processo que foi definido na organização. Para isso, deve-se garantir que todos os envolvidos conheçam e utilizem o processo e, sejam coletados dados que dêem informações sobre os resultados obtidos.

Após o guia do processo estar definido e revisado é feito um planejamento de como será avaliado o processo durante a sua execução. Neste passo, são definidas medidas que devem ser coletadas durante o uso do processo, visando a análise das metas que foram definidas na fase de análise estratégica. Para isso, pode ser utilizado, por exemplo, o método GQM [4]. Finalizado o planejamento da avaliação, o escopo de utilização do processo é definido. Esse passo é executado pelo engenheiro de processo e o RO e define se o guia do processo será utilizado inicialmente em todos os projetos da empresa, ou então, será executado apenas em um projeto piloto para avaliação. Na prática, temos observado que em MPEs, geralmente, não há muita diferença entre estes dois caminhos, em virtude do tamanho da organização. O próximo passo dessa etapa é treinar e motivar os participantes do processo, dentro do escopo que foi definido, para que estejam aptos a desempenhar suas atividades. Ao seu término, se inicia o uso do guia. Durante o período de uso do guia de processo, dúvidas que surgem são 
esclarecidas, sugestões e observações são anotadas e as medidas definidas durante o passo de planejamento da avaliação são coletadas. Os dados coletados são analisados $e$ interpretados pelo engenheiro de processo em cooperação com o RO. Os resultados dessa análise são documentados e apresentados para a diretoria, marcando a finalização de um ciclo de melhoria da abordagem. A finalização da abordagem faz parte do processo de gerência da abordagem.

\subsection{Gerência da Abordagem}

Todo o processo de estabelecimento de processo de software deve ser gerenciado, com o objetivo de planejar, monitorar, controlar e finalizar a sua execução. As atividades gerenciais incluem:

- Planejamento: planejar todo o processo de estabelecimento de processo de software, identificando as atividades que serão executadas e, principalmente, definindo cronograma, alocação de recursos, orçamento e riscos.

- Monitoria e Controle: visa acompanhar a execução da abordagem, principalmente em relação a esforço, tempo e riscos. Para isto, devem ser coletados e analisados dados. Ações corretivas devem ser iniciadas quando for necessário.

- Finalização: tem como objetivo finalizar um ciclo de melhorias realizado na empresa. Para isso, experiências são capturadas e documentadas, bem como benefícios adquiridos e problemas enfrentados. É importante que os dados obtidos sejam divulgados para toda a empresa com o objetivo de manter os envolvidos motivados, demonstrar 0 comprometimento da diretoria com a qualidade e disseminar uma cultura voltada para melhoria de processos. Isso permite ainda, prover uma aprendizagem contínua de todos.

A gerência é realizada pelo engenheiro de processo e pelo RO em interação com a diretoria e com os participantes do processo.

\subsection{Diretrizes gerais e de Adaptação}

A abordagem descreve atividades genéricas para o estabelecimento de processos em MPEs. No entanto, a sua aplicação em uma empresa específica requer, muitas vezes, adaptações e o seguimento de algumas diretrizes básicas. Por exemplo, as diretrizes a seguir devem ser observadas durante a aplicação da abordagem:

- Na execução da primeira fase da abordagem, os resultados de uma avaliação feita recentemente podem ser aproveitados. Caso as informações requeridas possam ser todas extraídas dessa avaliação, basta ao engenheiro do processo revisar seus resultados e buscar conhecer o cotidiano da empresa;

- Durante a fase de definição do processo é importante ter o suporte de uma ferramenta que gere um EPG, pois isso facilita bastante a disseminação do processo que está sendo definido, a sua melhoria (refinamentos) e a sua posterior implantação;

Com base na literatura e nas nossas experiências estamos continuamente identificando estas diretrizes, facilitando a aplicação da abordagem na prática. 


\section{Simpósio Brasileiro de Qualidade de Software}

\section{Primeiras Experiências}

Parte da concepção e uma primeira validação da abordagem estão sendo feitas baseadas, principalmente, em dois estudos de casos realizados em MPEs de Florianópolis/SC. As experiências adquiridas e os resultados obtidos são descritas nas seções a seguir.

\subsection{Estudo de Caso 1}

A primeira experiência de modelagem foi realizada numa pequena empresa voltada para o desenvolvimento de sistemas integrados de gestão para a indústria metal-mecânica e eletroeletrônica, tendo como principais campos de atuação os mercados de São Paulo, Rio de Janeiro, Paraná, Santa Catarina e Rio Grande do Sul. Atuando há mais de dez anos, a empresa vinha buscando melhorar a qualidade dos seus processos. Neste sentido, em abril de 2003, iniciou um programa de melhoria em parceria com o LQPS/UNIVALI, seguindo a versão da abordagem, que estava sendo desenvolvida em paralelo. Nesse estudo de caso, um dos colaboradores da empresa, que também era pesquisador do LQPS, foi alocado e treinado como engenheiro do processo. Além dele, um outro colaborador foi alocado para auxiliar na execução do programa e um pesquisador sênior do LQPS foi o responsável em dar orientação na aplicação da abordagem e na implantação do programa de melhoria.

$\mathrm{Na}$ fase de diagnóstico foi feita uma avaliação, conduzida pelo LQPS/UNIVALI, contextualizando a empresa e avaliando, em detalhes, quatro processos até o terceiro nível de capacidade, utilizando o método MARES. Na fase de análise estratégica, foi decido que os processos de fornecimento, suporte e desenvolvimento seriam os processos a serem estabelecidos, por se tratarem dos processos mais críticos para a empresa. Na fase de definição, foi gerado inicialmente um modelo descritivo para cada processo e, posteriormente, adicionadas algumas prescrições baseadas em best practices da engenharia de software. A seguir, foi realizada a documentação do modelo do processo, detalhando as atividades, seus critérios de entrada e saída, bem como os artefatos produzidos e consumidos. É importante ressaltar que a modelagem foi realizada por um funcionário da empresa, capacitado e apoiado pelo LQPS/UNIVALI, o qual também prestou consultoria na revisão periódica do modelo desenvolvido. A documentação do modelo foi sendo revisada e refinada pelos executores do processo. Além disso, novos elementos foram sendo adicionados, de modo que após oito meses, a empresa tinha a primeira versão do guia do processo. Ainda durante o ano de 2004, foram realizadas reavaliações do guia, gerando três atualizações até o momento. Para a fase de implantação, foi desenvolvido um Electronic Process Guide [8] [9] para suportar a documentação e manutenção do guia de processo, disponibilizando-o na Intranet da empresa.

\section{Custos}

O total de esforço empregado no estabelecimento do processo foi de aproximadamente 430 homens/hora, distribuídas num período de oito meses. Desse total, 69\% do esforço foram consumidos por colaboradores da empresa (representado por engenheiro de processo, funcionários/responsáveis, etc.). O restante, ou seja, 31\% foram consumidos por consultores externos. Tomando-se por base a capacidade produtiva total da empresa, o esforço empregado no estabelecimento de processos comprometeu, 


\section{Simpósio Brasileiro de Qualidade de Software}

aproximadamente $2 \%$ da sua capacidade produtiva total durante os 8 meses, o que caracteriza um custo baixo para a organização.

\section{Benefícios}

Após 12 meses de uso do modelo, desde a elaboração da primeira versão do guia, já podem ser observadas as primeiras indicações dos benefícios obtidos. Como resultado de uma pesquisa, envolvendo os nove colaboradores e a diretoria da empresa, realizada após a conclusão de um projeto piloto, observamos que, em geral, o estabelecimento de processos foi considerado benéfico. A maioria dos entrevistados já percebeu uma melhoria da qualidade do processo e do produto com a implantação do guia. Alguns resultados observados pela direitoria incluem:

- Redução de 50\% no número de service-packs (pacotes de correção fornecidos após a publicação da versão, normalmente para a correção de erros);

- Melhoria na determinação de valor de venda da implantação dos produtos desenvolvidos, a partir da identificação do esforço necessário estimado para cada atividade;

- Considerável melhoria na precisão das estimativas de esforço e prazo para os projetos de desenvolvimentos posteriores à aplicação do modelo de processos;

- Maior facilidade na disponibilização e difusão do conhecimento existente na organização.

Devido ao grande esforço consumido com aprendizagem, pelo funcionário que exerceu o papel de engenheiro de processo (120,4 homens-hora), inferimos que a melhor opção é o trabalho conjunto entre um consultor externo e um representante da organização. Sendo o último, alocado parcialmente para a aplicação da abordagem e treinado como engenheiro de processo, visando adquirir competência necessária para a continuidade das ações de melhoria. Tal experiência foi inserida na abordagem.

\subsection{Estudo de Caso 2}

O segundo estudo de caso foi realizado numa pequena empresa voltada para o desenvolvimento de produtos e serviços na área de ensino, treinamento e capacitação profissional. Criada há cinco anos, a empresa já mostrou ser viável, tendo como clientes o Boticário, SEBRAE, SENAI e GVT. Atualmente 13 pessoas fazem parte do seu quadro de colaboradores, incluindo os dois sócios diretores.

Da mesma forma que a empresa anterior, havia um interesse da diretoria em melhorar a qualidade dos seus produtos e serviços. Para isso, em maio de 2004 se iniciou o estabelecimento de processo de software seguindo nossa abordagem. $\mathrm{O}$ primeiro passo foi explicar a abordagem para a diretoria e obter o seu comprometimento. Depois disso, foi escolhido o representante da organização (RO) e explicado a ele suas responsabilidades. Um dos pesquisadores do LQPS/grupoQS, atuou como engenheiro de processo. Para a execução da fase de diagnóstico, foram aproveitados os resultados de uma avaliação do processo de software realizada no final de 2003 pelo LQPS/UNIVALI, utilizando o método MARES. Assim, o engenheiro de processo, em conjunto com o RO e com a diretoria, revisou os resultados obtidos, buscando identificar desvios que pudessem ter ocorrido nos cinco meses que haviam se passado desde a avaliação. Além disso, o engenheiro buscou conhecer melhor a cultura da empresa, suas políticas e suas metodologias de trabalho. O término dessa fase se deu 
em setembro de 2004 e, durante sua execução, a primeira versão da abordagem estava sendo concluída. Na segunda fase da abordagem, o processo de gerência de pedidos de alteração foi escolhido como o processo a ser estabelecido. Essa definição foi feita através de reuniões entre o engenheiro de processo, a diretoria e o RO. Além disso, um conjunto de ações foi planejado para a seqüência da aplicação da abordagem.

No começo de outubro de 2004, a terceira fase foi iniciada. A primeira atividade foi modelar o processo selecionado. Durante essa ação, o engenheiro de processo e o RO conceberam um esboço inicial do modelo e depois, foram refinando-o através de reuniões de brainstorming com os envolvidos no processo até chegar a primeira versão do modelo. Para dar agilidade ao processo e garantir o histórico dos pedidos de alteração feitos pelos clientes, foi necessário a adoção de uma ferramenta para dar o suporte necessário ao processo. A empresa resolveu adotar uma ferramenta gratuita para esse propósito, a qual foi feita algumas pequenas customizações. Além disso, foi necessário dar toda a orientação aos envolvidos sobre como desempenhar as atividades descritas no modelo do processo, utilizando a ferramenta. Para facilitar essa orientação, foi construído um guia do processo, que foi disponibilizado num servidor de arquivos da empresa. A partir daí houve vários refinamentos no guia, de modo que a fase de implantação do processo iniciou-se no final de novembro do mesmo ano. Durante essa fase, ocorreu um treinamento com todos os envolvidos. Além disso, foi feito um planejamento de como seria avaliado o processo implantado e, uma data foi oficializada para marcar o início do uso do guia. A implantação do processo foi efetivada em janeiro de 2005 e, desde então, estamos avaliando os resultados obtidos. Na segunda semana de fevereiro foi aplicado um questionário junto aos envolvidos que nos dão algumas informações iniciais dos resultados obtidos.

\section{Custos}

Para o estabelecimento do processo de gerência de pedidos de alteração, que conta atualmente, com nove atividades e seis papéis definidos, foram necessários 186 homens-hora, distribuídos em 14 meses. A tabela 1, a seguir, apresentada o esforço consumido em cada uma das fases da abordagem. Tais dados foram considerados aceitáveis pela diretoria da empresa.

Tabela 1: Distribuição de esforço por Fase

\begin{tabular}{|c|c|c|c|}
\hline Fase/ Homem-horas & Engenheiro do Processo & Organização & Total: \\
\hline Diagnóstico & 35,5 & 30,5 & 66 \\
\hline Análise Estratégica & 3 & 2 & 5 \\
\hline Definição & 20,5 & 56,5 & 77 \\
\hline Implantação & 7,5 & 7,5 & 15 \\
\hline Gerência & 11,5 & 11,5 & 23 \\
\hline Total: & 78 & 108 & 186 \\
\hline
\end{tabular}

\section{Benefícios}

Apresar do curto período de utilização do processo, já observamos que o guia desenvolvido descreve adequadamente o processo e que está sendo efetivamente utilizado pelos seus atores, o que pode ser verificado através da ferramenta utilizada para controle dos pedidos. 
Além disso, apesar de o processo ter sido implantado há pouco tempo, alguns benefícios já foram sentidos pelos envolvidos:

- Melhoria na compreensão do processo e aumento da clareza nos papéis e nas responsabilidades de cada um;

- Redução do tempo na busca de informações sobre um pedido específico, segundo a opinião da maioria dos envolvidos;

- Auxílio na execução e gerência das atividades, provido pelo registro das atividades na ferramenta utilizada, pelas medidas coletadas e pelo detalhamento de como as atividades devem ser executadas;

- Definição e coleta de medidas que permitem mensurar a eficiência operacional, como, por exemplo: número de pedidos atendidos por período e número de pedidos de alteração por produto, informações que anteriormente não estavam disponíveis;

- Aumento da satisfação dos clientes e vantagem competitiva. Segundo um dos diretores, alguns clientes se mostram surpresos pelo fato da empresa ter o processo de gerência de pedidos de alteração estabelecido.

No entanto, durante o uso do guia com o processo estabelecido, já foram identificados alguns pontos de melhoria, como, por exemplo, a falta de algumas atividades e de alguns templates de documentos. Nesse sentido, já foi iniciado um ciclo de melhoria do processo, prevista para ser finalizada em maio de 2005.

\section{Conclusões}

Neste artigo propomos uma abordagem para estabelecimento de processos de software em micro e pequenas empresas. Apesar da abordagem ainda estar em fase de validação e sendo aperfeiçoada com base nas experiências adquiridas, os primeiros resultados indicam que ela pode ser aplicada de forma efetiva e eficiente para definir e implantar processos de software neste tipo de empresa. Os principais diferenciais em relação a outras abordagens existentes são o custo baixo, a flexibilidade, o direcionamento para MPEs e o grau de detalhamento no processo a ser realizado. Como atividades futuras estão agendadas novos estudos de caso para continuar a validar e a evoluir a abordagem.

\section{Referências}

[1] ACUÑA, S.T.; ANTONIO, A. de; FERRÉ, X.; LÓPEZ, M.; MATÉ, L. The Software process: modeling, evaluation and improvement. Handbook of Software Engineering and Knowledge Engineering, 2000.

[2] ANACleto, A.; VON WANGENHEIM, C.G.; SALVIANO, C. F.; SAVI, R.. A Method for Process Assessment in Small Software Companies. $4^{\text {th }}$ International SPICE Conference on Process Assessment and Improvement, Portugal, 2004.

[3] ANACleto, A.; VON WANGEnHEIM, C. G.; SALVIANO, C. F. Um Método de Avaliação de Processos de Software em Micro e Pequenas Empresas. IV Simpósio Brasileiro de Qualidade de Software, Porto Alegre, 2005.

[4] BASILI, V. R.; CALDIERA, G.; ROMBACH, H. D. Goal/Question/Metric Approach. In: MARCINIAK, J. J. Encyclopedia of Software Engineering, vol. 1. John Wiley \& Sons, 1994. 
[5] BECKER, U.; HAMANN, D.; VERLAGE, M. Descriptive Modelling of Software Processes. IESE-Report, Alemanha, Dec.1997, 15 p.

[6] CHRISTIE, A. M. et al. Software Process Automation: Interviews, Survey and Workshop Results. (CMU/SEI-97-TR- 008) Pittsburgh: PA: Software Engineering Inst., Carnegie Mellon Univ., October 1997.

[7] DINGsøYR, T.; MOE, N.B.; DYBA, T.; CONRADI, R. A Workshop-Oriented Approach for Defining Electronic Process Guides. In: JURISTO, N.; ACUÑA, S.T. Software Process Modelling, Kluwer Academic Publishers, 2005, p. 187-205.

[8] HAUCK, J. C. R. Desenvolvimento de um sistema de software para gerência de manuais de processo de software em micro e pequenas empresas. Trabalho de Conclusão do Curso. UNIVALI - Universidade do Vale do Itajaí, São José, 2004.

[9] HAUCK, J. C. R.; VON WANGenheiM, C. G. Modelando o Processo de Software em uma Pequena Empresa: O Caso VOID CAZ. VI Simpósio Internacional de Melhoria de Processo de Software - SIMPROS, São Paulo, 2004.

[10] INSTITUT EXPERIMENTELLES SOFTWARE ENGINEERING. SPEARMINT. Disponível em: http://www.iese.fhg.de/Spearmint_EPG. Acesso em: 28 fev. 2004.

[11] INSTITUTE OF ELECTRICAL AND ELECTRONICS ENGINEERS. IEEESTD-610: Standard Computer Dictionary: A Compilation of IEEE Standard Computer Glossaries. IEEE, 1990.

[12] INTERNATIONAL ORGANIZATION FOR STANDARDIZATION. ISO/IEC 12207:1995/Amd 1:2002: Information technology -- Software life cycle processes. ISO/IEC International Standard, 2002.

[13] INTERNATIONAL ORGANIZATION FOR STANDARDIZATION. ISO/IEC 15504: Information Technology Process Assessment, Part 1 to Part 5. ISO/IEC International Standard, 2003-2005 (in development)

[14] KELLNER, M.I.; BECKER-KORNSTAEDT, U.; RIDDLE, W.E.; TOMAL, J.; VERLAGE, M. Process Guides: Effective guidance for Process Participants. $5^{\text {th }}$ International Conference on the Software Process, Illinois, USA, Jun. 1998.

[15] KYLE, B. SWOT Analysis - Beyond the Text Book. Disponível em: http://www.websitemarketingplan.com/Arts/SWOT.htm. Acesso em: 18 mar. 2004.

[16] LONCHAMP, J. A Structured conceptual and terminological framework for software process engineering. [S.l.]: IEEE, 1993.

[17] MACHADO, L.F.D.C. Modelo para Definição de Processos de Software na Estação TABA. Dissertação de Mestrado. COPPE/UFRJ. Rio de Janeiro, mar. 2001.

[18] MACHADO, L.F.D.C. et. al. Experiência na Definição e Implantação de Processos de Software. Workshop de Qualidade de Software - WQS, 2001.

[19] MINISTÉRIO DA CIÊNCIA E TECNOLOGIA. Qualidade e Produtividade no Setor de Software Brasileiro: Resultados da Pesquisa 2001. Disponível em: http://www.mct.gov.br/Temas/info/Dsi/Quali2001/Public2001.htm Acesso em: 28 fev. 2004. 
[20] MOE, N.B.; DINGSØYR, T.; DYBA, T.; JOHANSEN, T. Process Guides as Software Process Improvement in a Small Company. Proceedings of the European Software Process Improvement Conference, Nürnberg, Set. 2002.

[21] PAULK, M. C.; WEBER, C. V.; CURTIS, B.; CHRISSIS, M. B. The Capability Maturity Model: Guidelines for Improving the Software Process. Addison-Wesley, 1994.

[22] RATIONAL SOFTWARE CORPORATION. Rational Unified Process. Disponível em: http://www.rational.com/products/rup/ Acesso em: 10 fev. 2005.

[23] ROCHA, A. R. C. et al. Experiência em Definição, Uso e Melhoria de Processos de Software. In: ROCHA, A. R. C da; MALDONADO, J. C.; WEBER, K. C. Qualidade de Software: Teoria e Prática. São Paulo: Prentice Hall, 2001, p.185-191.

[24] SCOTT, L., ZETTEL, J., HAMANN, D. Suporting Process Engineering in Pactice: An Experience Based Scenario. Technical Report IESE No. 033.00/E, Fraunhofer IESE, Alemanha, 2000

[25] SCOTT, L.; KURNIAWATI, F. WAGNER - Web-based process Guide and Experience Repository. Centre for Advanced Software Engineering Research (CAESER) - University of New South Wales, Sydney, 2001.

[26] SCOTT, L.; CARVALHO, L; JEFFERY, R. A Process-Centred Experience Repository for a Small Software Organisation. In: Proceedings of the 2002 AsiaPacific Software Engineering Conference, pp 603-609.

[27] SCOTT, L.; CARVALHO, L; JEFFERY, R.; D’AMBRA, J.; BECKERKORNSTAEDT, U. Understanding the use of an Electronic Process guide. Centre for Advanced Software Engineering Research (CAESER), University of New South Wales, Sydney, 2002.

[28] SERVIÇO BRASILEIRO DE APOIO ÀS MICRO E PEQUENAS EMPRESAS. Fatores Condicionantes e Taxa de Mortalidade de Empresas no Brasil. Relatório de Pesquisa. Brasília, ago. 2004.

[29] SOFTWARE ENGINEERING INSTITUTE. Capability Maturity Model ${ }^{\circledR}$ Integration (CMMI $\left.\mathbf{S M}_{\mathbf{S M}}\right)$ Continuous Representation. Disponível em: http://www.sei.cmu.edu/cmmi/. Acessado em: 20 fev. 2005.

[30] SOUZA, M. B. de. Modelo de processo de software: aplicação em uma empresa júnior. Trabalho de Conclusão de Curso. INE/UFSC. Florianópolis, jan. 2004.

[31] WEBER, S. Um estudo de Caso para Modelagem de Processos em Micro e Pequenas Empresas de Software. Trabalho de Conclusão de Curso. INE/UFSC. Florianópolis, set. 2002.

[32] ZAHRAN, S. Software Process Improvement: Practical Guidelines for Business Success. Edinburgh: Addison-Wesley, 1998. ISBN 0-201-17782-X. 